\title{
THE DUAL OF THE SPACE OF REGULATED FUNCTIONS IN SEVERAL VARIABLES
}

\author{
YUNYUN YANG AND RICARDO ESTRADA
}

\begin{abstract}
We consider a class of regulated functions of several variables, namely, the class of functions $\phi$ defined in an open set $U \subset \mathbb{R}^{n}$ such that at each $\mathbf{x}_{0} \in U$ the "thick" limit$$
\phi_{\mathbf{x}_{0}}(\mathbf{w})=\lim _{\varepsilon \rightarrow 0^{+}} \phi\left(\mathbf{x}_{0}+\varepsilon \mathbf{w}\right),
$$

exists uniformly on $\mathbf{w} \in \mathbb{S}$, the unit sphere of $\mathbb{R}^{n}$, and such that $\phi_{\mathbf{x}_{0}}$ is a continuous function of $\mathbf{w}$ at each $\mathbf{x}_{0} \in U$.

We identify the elements of the dual spaces of several Banach space of such regulated functions in several variables as signed measures with absolutely convergent sums of "thick" delta functions concentrated at a countable set.
\end{abstract}

\section{INTRODUCTION}

Regulated functions in one variable are those functions whose lateral limits exist at every point [4]. In [5] a natural generalization to several variables was introduced, namely, regulated functions $\phi$ in an open set $U \subset \mathbb{R}^{n}$ are those for which for each $\mathbf{x}_{0} \in U$ the limit

$$
\phi_{\mathbf{x}_{0}}(\mathbf{w})=\lim _{\varepsilon \rightarrow 0^{+}} \phi\left(\mathbf{x}_{0}+\varepsilon \mathbf{w}\right),
$$

exists uniformly for $\mathbf{w} \in \mathbb{S}$, the unit sphere of $\mathbb{R}^{n}$. We call the function $\phi_{\mathbf{x}_{0}}(\mathbf{w})$ the "thick" limit of $\phi$ at the point $\mathbf{x}_{0}$, due to its relation to the thick points introduced in [6].

We shall also ask that the thick limits of the regulated function $\phi$ to be continuous functions of $\mathbf{w} \in \mathbb{S}$. It was shown in [5] that if the thick limits $\phi_{\mathbf{x}_{0}}(\mathbf{w})$ are continuous functions of $\mathbf{w}$ at each $\mathbf{x}_{0} \in U$ then the set of singularities $\mathfrak{S}$, the set of points where the ordinary limit does not exist, is countable: $|\mathfrak{S}| \leq \aleph_{0}$. Actually $|\mathfrak{S}| \leq \aleph_{0}$ even if distributional regulated

2010 Mathematics Subject Classification. 28C05, 26B05, $26 \mathrm{~B} 35$.

Key words and phrases. Regulated functions, dual spaces, signed measures, thick deltas.

The authors gratefully acknowledge support from NSF, through grant number 0968448 . 
functions $[15,16]$ in several variables are considered as long as the thick limits are continuous.

We must point out that this not the only possible definition of regulated functions in several variables. There are other characterizations of regulated functions in one variable, namely as the uniform limit of step functions [4], or as functions whose average variation is zero [1]. Therefore other possible generalizations of the notion of regulated function to several variables [3] and actually to general topological spaces [9] have been considered. Generalizations obtained through the theory of integration [2] seem also possible.

However, considering functions that have continuous thick limits allows us to construct interesting Banach spaces of regulated functions, and, more generally, topological vector spaces of regulated functions whose dual spaces can be described in a natural way, similar to the description of Radon signed measures. Indeed, the ordinary Dirac delta function is a Radon signed measure, and each Radon signed measure is the sum of a continuous part plus a discrete part, a sum of Dirac delta functions. We show that in spaces of regulated functions there is the notion of thick delta function and that each element of the dual space is the sum of a continuous part plus a discrete part, a sum of thick Dirac delta functions.

The dual spaces of several Banach spaces of regulated functions in one variable have been given by various authors, who have used different integrals to represent the continuous linear functionals. Kaltenborn [8], in 1934, found the duals of the spaces of normalized and the space of all regulated functions defined in a compact interval $[a, b]$ by using the Dushnik interior integral. Hildebrandt [7] considered the dual of spaces of regulated functions in $\mathbb{R}$. In several articles, Tvrdý $[12,13,14]$ gave an alternative description of the space of left continuous regulated functions in a compact interval $[a, b]$ by using Perron-Stieltjes integrals. Talvila $[10,11]$ has employed his distributional integrals and those descriptions to find the dual of other interesting spaces. Our results also apply to the case of one variable, of course, and thus a new, equivalent description of dual spaces in one variable is obtained.

In order to consider Banach spaces of regulated functions, we need to discuss a simple but annoying situation. Indeed, shall we consider regulated functions as functions defined at all points?, or, shall we consider their equivalence class in the almost everywhere sense? The problem is that when one uses the supremum norm then functions equal to zero a.e. do not have null length, as is the case in the spaces $L^{p}$ for $p<\infty$. Let $\mathcal{B}_{\text {meas }}[a, b]$ be the space of bounded, Lebesgue measurable functions defined in $[a, b]$, with the supremum norm, and let $L^{\infty}[a, b]$ be the usual Lebesgue space of equivalence classes of bounded measurable functions, equal a.e., with the essential supremum norm. Then there is a natural projection $\pi: \mathcal{B}_{\text {meas }}[a, b] \rightarrow L^{\infty}[a, b]$, 
but the two spaces are not the same. The characteristic function of a set of null measure, $\chi_{Z}$, has norm 1 in $\mathcal{B}_{\text {meas }}[a, b]$, even though $\pi\left(\chi_{Z}\right)=0$. What this means is that we shall obtain two types of spaces of regulated functions in one or several variables: one space, $\mathcal{R}_{\mathrm{t}}$, contains the totality of all regulated functions, while another space, $\mathcal{R}$, contains only functions normalized in some fashion, and corresponds to the idea of working in a subspace of $L^{\infty}$.

The plan of the article is as follows. In Section 2, for an open bounded set $U$ we introduce the Banach spaces $\mathcal{R}_{\mathrm{t}}[U]$ and $\mathcal{R}[U]$ of all and of normalized regulated functions in $\mathbb{R}^{n}$ that vanish outside of $\bar{U}$. In Section 3 we define the notion of thick delta functions and prove that if the support of an element of $\mathcal{R}^{\prime}[U]$ is a single point, then it must be a thick delta. We also prove that the set of regulated functions with a finite number of discontinuities is dense in $\mathcal{R}_{\mathrm{t}}[U]$ and, correspondingly, in $\mathcal{R}[U]$. The elements of the dual spaces $\mathcal{R}_{\mathrm{t}}^{\prime}[U]$ and $\mathcal{R}^{\prime}[U]$ are then described as the sum of a continuous part plus a discrete part, a sum of thick Dirac delta functions. In Section 4 we consider spaces of regulated functions over a general open set, spaces that are no longer Banach spaces, and describe their duals. Our results also apply in one dimension, thus in Section 5 we describe the linear functionals in spaces of regulated functions in one variable and compare these results with the known description of such functionals available in the literature.

\section{Regulated FunCtions in SEVERAl VARiables}

We first consider regulated functions in several variables [5].

Let $\mathcal{X}$ be a topological vector space of functions defined in the unit sphere $\mathbb{S}$ of $\mathbb{R}^{n}$. For example, $\mathcal{X}$ could be $C(\mathbb{S})$, the continuous functions on $\mathbb{S}$, or the Lebesgue spaces $L^{p}(\mathbb{S})$, or $\mathcal{B}_{s}(\mathbb{S})$, the space of bounded functions in $\mathbb{S}$ with the topology of uniform convergence, or $\mathcal{B}_{w}(\mathbb{S})$, the space of bounded functions in $\mathbb{S}$ with the topology of pointwise convergence.

Definition 2.1. Let $\phi$ be a function defined in a region $U$ of $\mathbb{R}^{n}$. We say that $\phi$ is $\mathcal{X}$-regulated if for each $\mathbf{x}_{0} \in U$ there exists $r\left(\mathbf{x}_{0}\right)>0$, such that if $0<\varepsilon<r\left(\mathbf{x}_{0}\right)$, then the function $\phi\left(\mathbf{x}_{0}+\varepsilon \mathbf{w}\right), \mathbf{w} \in \mathbb{S}$, belongs to $\mathcal{X}$, and the limit

$$
\phi_{\mathbf{x}_{0}}(\mathbf{w})=\lim _{\varepsilon \rightarrow 0^{+}} \phi\left(\mathbf{x}_{0}+\varepsilon \mathbf{w}\right)
$$

exists in $\mathcal{X}$.

If the function $\phi_{\mathbf{x}_{0}}(\mathbf{w}), \mathbf{w} \in \mathbb{S}$, is not a constant, then we call it the thick limit of $f$ at the point $\mathbf{x}_{0}$. Otherwise if $\phi_{\mathbf{x}_{0}}(\mathbf{w}) \equiv \phi_{\mathbf{x}_{0}}$, a constant, then we call it an ordinary limit at the point $\mathbf{x}_{0}$; if $\phi\left(\mathbf{x}_{0}\right)=\phi_{\mathbf{x}_{0}}$ then $\phi$ would be

continuous, in some sense that depends on $\mathcal{X}$, at the point $\mathbf{x}_{0}$ and naturally, 
in many cases, one can redefine the function $\phi$ at $\mathbf{x}_{0}$ to make it continuous at $\mathbf{x}_{0}$ if the ordinary limit exists.

Observe that when $n=1$ then $\mathbb{S}=\{-1,1\}$, so that the space of all functions defined in $\mathbb{S}$ is basically $\mathbb{R}^{\mathbb{S}} \simeq \mathbb{R}^{2}$. Since all Haussdorf vector topologies in $\mathbb{R}^{2}$ are equivalent, there is just one space $\mathcal{X}$ in this case, and for this space, $\mathcal{X}$-regulated means regulated, namely, it means that the limits from the right and left exist at each point.

We shall consider $\mathcal{X}$-regulated functions where $\mathcal{X}=\mathcal{B}_{s}(\mathbb{S})$, the space of bounded functions in $\mathbb{S}$ with the supremum norm.

Definition 2.2. Let $U$ be an open bounded set in $\mathbb{R}^{n}$. The space of regulated functions $\mathcal{R}_{\mathrm{t}}[U]$ is the space of all $\mathcal{B}_{s}(\mathbb{S})$-regulated functions defined in all $\mathbb{R}^{n}$, that vanish in $\mathbb{R}^{n} \backslash \bar{U}$, and such that the thick limits $\phi_{\mathbf{x}_{0}}$ are continuous functions in $\mathbb{S}$ for each $\mathbf{x}_{0} \in \mathbb{R}^{n}$.

Observe that if $\phi \in \mathcal{R}_{\mathrm{t}}[U]$ then for a given $\varepsilon$ the function $\phi\left(\mathbf{x}_{0}+\varepsilon \mathbf{w}\right)$ may or may not be continuous, but the limit of such functions as $\varepsilon \rightarrow 0$, $\phi_{\mathbf{x}_{0}}$ must be, that is, $\phi_{\mathbf{x}_{0}} \in C(\mathbb{S})$. It is known that if the thick limits are continuous, then the set of points where $\phi_{\mathbf{x}_{0}}$ is not constant is countable at the most [5].

Definition 2.3. A regulated function $\phi \in \mathcal{R}_{\mathrm{t}}[U]$ is normalized if

$$
\phi\left(\mathbf{x}_{0}\right)=\frac{1}{c_{n}} \int_{\mathbb{S}} \phi_{\mathbf{x}_{0}}(\mathbf{w}) \mathrm{d} \sigma(\mathbf{w}),
$$

for each $\mathbf{x}_{0} \in \mathbb{R}^{n}$, where $c_{n}=\int_{\mathbb{S}} \mathrm{d} \sigma(\mathbf{w})$ is the $(n-1)$-measure of the unit sphere $\mathbb{S}$. The space $\mathcal{R}[U]$ is the subspace of $\mathcal{R}_{\mathrm{t}}[U]$ formed by the normalized regulated functions.

We shall now establish that $\mathcal{R}[U]$ and $\mathcal{R}_{\mathrm{t}}[U]$ are Banach spaces with the supremum norm.

Proposition 2.4. Any function $\phi \in \mathcal{R}_{\mathrm{t}}[U]$ is bounded in $\mathbb{R}^{n}$.

Proof. If $\phi$ were not bounded, we would be able to find a sequence $\left\{\mathbf{x}_{n}\right\}_{n=1}^{\infty}$ of points where $\left|\phi\left(\mathbf{x}_{n}\right)\right| \geq n$. Clearly all the points $\mathbf{x}_{n}$ belong to the compact space $\bar{U}$, and thus the sequence has a convergent subsequence, which for simplicity we may assume is $\left\{\mathbf{x}_{n}\right\}_{n=1}^{\infty}$ itself, $\mathbf{x}_{n} \rightarrow \mathbf{x}^{*}$. Hence $\sup _{0<\left|\mathbf{x}-\mathbf{x}^{*}\right|<r}|\phi(\mathbf{x})|$ $=\infty$ for all $r>0$.

However, $\phi$ is $\mathcal{B}_{s}(\mathbb{S})$-regulated at $\mathbf{x}^{*}$, and thus there exists $r_{0}>0$ such that

$$
\max _{\mathbf{w} \in \mathbb{S}}\left|\phi_{\mathbf{x}^{*}}(\mathbf{w})-\phi\left(\mathbf{x}^{*}+\varepsilon \mathbf{w}\right)\right| \leq 1
$$

for $0<\varepsilon<r_{0}$. But (2.3) implies that $|\phi(\mathbf{x})| \leq M$ for $0<\left|\mathbf{x}-\mathbf{x}^{*}\right|<r_{0}$, where $M=\left\|\phi_{\mathbf{x}^{*}}\right\|_{\text {sup }}+1$. 
It is interesting that if $\mathcal{X} \neq \mathcal{B}_{s}(\mathbb{S})$ then $\mathcal{X}$-regulated functions do not have to be bounded. For example, if $p<\infty$, there are functions which are $L^{p}(\mathbb{S})$-regulated at each point but unbounded.

It follows from the Proposition 2.4 that $\mathcal{R}_{\mathrm{t}}[U] \subset \mathcal{B}_{s}\left(\mathbb{R}^{n}\right)$. Therefore with the supremum norm $\mathcal{R}_{\mathrm{t}}[U]$ becomes a normed space.

Proposition 2.5. $\mathcal{R}_{\mathrm{t}}[U]$ is closed in $\mathcal{B}_{s}\left(\mathbb{R}^{n}\right)$, and hence a Banach space.

Proof. Let $\left\{\phi_{n}\right\}_{n=1}^{\infty}$ be a sequence of $\mathcal{R}_{\mathrm{t}}[U]$ that converges uniformly in $\mathbb{R}^{n}$ to $\phi \in \mathcal{B}_{s}\left(\mathbb{R}^{n}\right)$; we need to show that $\phi \in \mathcal{R}_{\mathrm{t}}[U]$. Since it is clear that $\phi$ vanishes outside of $\bar{U}$, what we need to show is that $\phi$ is $\mathcal{B}_{s}(\mathbb{S})$-regulated and that $\phi_{\mathbf{x}_{0}} \in C(\mathbb{S})$ for each $\mathbf{x}_{0} \in \mathbb{R}^{n}$.

Fix $\mathbf{x}_{0} \in \mathbb{R}^{n}$. Let $\eta>0$. There exists $n_{0} \in \mathbb{N}$ such that $\left\|\phi-\phi_{n}\right\|_{\text {sup }}<$ $\eta / 3$ for $n \geq n_{0}$. There exists $r_{0}>0$ such that if $0<\varepsilon_{1}, \varepsilon_{2}<r_{0}$ then $\left|\phi_{n_{0}}\left(\mathbf{x}_{0}+\varepsilon_{1} \mathbf{w}\right)-\phi_{n_{0}}\left(\mathbf{x}_{0}+\varepsilon_{2} \mathbf{w}\right)\right|<\eta / 3$. Hence

$$
\limsup _{\varepsilon_{1}, \varepsilon_{2} \rightarrow 0^{+}}\left(\max _{\mathbf{w} \in \mathbb{S}}\left|\phi\left(\mathbf{x}_{0}+\varepsilon_{1} \mathbf{w}\right)-\phi\left(\mathbf{x}_{0}+\varepsilon_{2} \mathbf{w}\right)\right|\right) \leq \eta,
$$

and it follows that $\phi_{\mathbf{x}_{0}}$, the limit of $\phi\left(\mathbf{x}_{0}+\varepsilon \mathbf{w}\right)$ as $\varepsilon \rightarrow 0^{+}$, exists in $\mathcal{B}_{s}(\mathbb{S})$. It is easy to see that $\left(\phi_{n}\right)_{\mathbf{x}_{0}}$ converges to $\phi_{\mathbf{x}_{0}}$ in $\mathcal{B}_{s}(\mathbb{S})$, and the continuity of the $\left(\phi_{n}\right)_{\mathbf{x}_{0}}$ yields that $\phi_{\mathbf{x}_{0}} \in C(\mathbb{S})$.

We can define a normalization operation in $\mathcal{R}_{\mathrm{t}}[U]$ as follows.

Definition 2.6. Let $\phi \in \mathcal{R}_{\mathrm{t}}[U]$. The function $\psi=N(\phi)$ is the normalization of $\phi$, given as

$$
\psi(\mathbf{x})=\frac{1}{c_{n}} \int_{\mathbb{S}} \phi_{\mathbf{x}}(\mathbf{w}) \mathrm{d} \sigma(\mathbf{w}), \quad \mathbf{x} \in \mathbb{R}^{n} .
$$

The normalization of a regulated function of the space $\mathcal{R}_{\mathrm{t}}[U]$ is also regulated, and in fact the normalized function coincides with the original function save on a countable set.

Proposition 2.7. If $\phi \in \mathcal{R}_{\mathrm{t}}[U]$ then $\psi=N(\phi)$ is $\mathcal{B}_{s}(\mathbb{S})$-regulated, and actually $\psi_{\mathbf{x}}=\phi_{\mathbf{x}}$ at each $\mathbf{x} \in \mathbb{R}^{n}$. The operator $N$ satisfies $N^{2}=N$ and is a projection from $\mathcal{R}_{\mathrm{t}}[U]$ to $\mathcal{R}[U]$. Hence $\mathcal{R}[U]$ is a Banach space.

Proof. Fix $\mathbf{x}_{0} \in \mathbb{R}^{n}$. Let $\eta>0$. There exists $\delta>0$ such that if $\mathbf{w}_{1}, \mathbf{w}_{2} \in \mathbb{S}$ satisfy $\left|\mathbf{w}_{1}-\mathbf{w}_{2}\right|<\delta$, then $\left|\phi_{\mathbf{x}_{0}}\left(\mathbf{w}_{1}\right)-\phi_{\mathbf{x}_{0}}\left(\mathbf{w}_{2}\right)\right|<\eta / 2$, and there exists $r_{0}$ such that if $0<\varepsilon<r_{0}$ then $\left|\phi_{\mathbf{x}_{0}}(\mathbf{w})-\phi\left(\mathbf{x}_{0}+\varepsilon \mathbf{w}\right)\right|<\eta / 2$ for all $\mathbf{w} \in \mathbb{S}$. Hence

$$
\left|\phi_{\mathbf{x}_{0}}(\mathbf{w})-\phi\left(\mathbf{x}_{0}+\varepsilon \mathbf{v}\right)\right|<\eta \quad \text { if }|\mathbf{w}-\mathbf{v}|<\delta \text { and } 0<\varepsilon<r_{0} .
$$

It follows that if $\rho>0$ is small enough, then for any $\mathbf{w} \in \mathbb{S}$,

$$
\left|\phi_{\mathbf{x}_{0}}(\mathbf{w})-\frac{1}{c_{n}} \int_{\mathbb{S}} \phi\left(\mathbf{x}_{0}+\varepsilon \mathbf{w}+\rho \mathbf{v}\right) \mathrm{d} \sigma(\mathbf{v})\right|<\eta
$$


and since $\int_{\mathbb{S}} \phi(\mathbf{x}+\rho \mathbf{v}) \mathrm{d} \sigma(\mathbf{v}) \rightarrow c_{n} \psi(\mathbf{x})$ as $\rho \rightarrow 0^{+}$for any $\mathbf{x}$,

$$
\left|\phi_{\mathbf{x}_{0}}(\mathbf{w})-\psi\left(\mathbf{x}_{0}+\varepsilon \mathbf{w}\right)\right| \leq \eta \quad \text { for } 0<\varepsilon<r_{0} .
$$

We thus obtain that $\psi$ is $\mathcal{B}_{s}(\mathbb{S})$-regulated and $\psi_{\mathbf{x}_{0}}=\phi_{\mathbf{x}_{0}}$.

The fact that $N$ is a projection from $\mathcal{R}_{\mathrm{t}}[U]$ to $\mathcal{R}[U]$ that satisfies $N^{2}=N$ is now clear.

The Proposition 2.7 allows to write

$$
\mathcal{R}_{\mathrm{t}}[U]=\mathcal{R}[U] \oplus \mathcal{R}_{\mathrm{n}}[U],
$$

where $\mathcal{R}_{\mathrm{n}}[U]=\operatorname{Ker} N$. A function $\phi \in \mathcal{R}_{\mathrm{t}}[U]$ belongs to $\mathcal{R}_{\mathrm{n}}[U]$ if and only if $\phi_{\mathbf{x}}=0$ for all $\mathbf{x} \in \mathbb{R}^{n}$. It is not hard to see that $\phi \in \mathcal{R}_{\mathrm{n}}[U]$ if and only if there exists a sequence of different points of $\bar{U},\left\{\mathbf{x}_{n}\right\}_{n=1}^{\infty}$, such that $\phi(\mathbf{x})=0$ if $\mathbf{x} \neq \mathbf{x}_{n}$ for all $n$, and $\lim _{n \rightarrow \infty} \phi\left(\mathbf{x}_{n}\right)=0$.

If $\pi: \mathcal{B}_{\text {meas }}[U] \rightarrow L^{\infty}[U]$ is the projection, then we have that $\pi\left(\mathcal{R}_{\mathrm{n}}[U]\right)=$ $\{0\}$, and thus

$$
\pi\left(\mathcal{R}_{\mathrm{t}}[U]\right)=\pi(\mathcal{R}[U]) .
$$

Therefore, the two Banach spaces $\mathcal{R}[U]$ and the subspace $\pi\left(\mathcal{R}_{\mathrm{t}}[U]\right)$ of $L^{\infty}[U]$ are isometric. This means that in a sense $\mathcal{R}[a, b]$ "is" the space of regulated functions in the context of $L^{\infty}[a, b]$.

\section{The dual spaces $\mathcal{R}_{t}^{\prime}[U]$ And $\mathcal{R}^{\prime}[U]$}

We shall now describe the dual spaces $\mathcal{R}_{\mathrm{t}}^{\prime}[U]$ and $\mathcal{R}^{\prime}[U]$. If $\mu$ belongs to the dual space of $\mathcal{R}[U]$ or of $\mathcal{R}_{\mathrm{t}}[U]$, we shall denote the evaluation of $\mu$ on a regulated function as $\langle\mu, \phi\rangle$, or as $\langle\mu(\mathbf{x}), \phi(\mathbf{x})\rangle$ when we want to clearly indicate the variable of evaluation. We will show that the elements of these dual spaces, $\mathcal{R}_{\mathrm{t}}^{\prime}[U]$ and $\mathcal{R}^{\prime}[U]$, are signed measures with a sum of "thick delta functions". In what follows we shall denote by $\mathcal{M}(X)=(C(X))^{\prime}$ the space of Radon measures on a compact space $X$.

We define $\mathcal{R}_{\mathrm{t}}([U] ; F)$ to be the set of regulated functions that are continuous in $\bar{U} \backslash F$, where $F \subset \bar{U}$ is a finite set of points. Respectively, we denote by $\mathcal{R}([U] ; F)$ the subset of normalized regulated functions of $\mathcal{R}_{\mathrm{t}}([U] ; F)$.

If $F=\emptyset$, then $\mathcal{R}_{\mathrm{t}}([U] ; \emptyset)$ is a closed subspace of $C(\bar{U})$. It is the space of continuous functions in $\bar{U}$ that vanish on the boundary. Hence, if $\mu_{\emptyset} \in$ $\mathcal{R}_{\mathrm{t}}([U] ; \emptyset)$, then $\mu_{\emptyset}$ gives a Radon measure in $U$, which can be written as

$$
\mu_{\emptyset}=\mu_{\mathrm{cont}}+\mu_{\mathrm{discr}}
$$

where $\mu_{\text {cont }}$ is the continuous part and where $\mu_{\text {discr }}$ is the discrete part,

$$
\mu_{\text {discr }}(\mathbf{x})=\sum_{n=1}^{\infty} \gamma_{n} \delta\left(\mathbf{x}-\mathbf{x}_{n}\right)
$$


a sum of Dirac delta functions at some points $\left\{\mathbf{x}_{n}\right\}_{n=1}^{\infty}$ of $U$ that satisfies $\sum_{n=1}^{\infty}\left|\gamma_{n}\right|<\infty$, since actually

$$
\left\|\mu_{\emptyset}\right\|=\int_{U} \mathrm{~d}\left|\mu_{\text {cont }}\right|+\sum_{n=1}^{\infty}\left|\gamma_{n}\right| .
$$

Here $\left\|\mu_{\emptyset}\right\|=\sup \left\{\left\langle\mu_{\emptyset}, \phi\right\rangle: \phi \in \mathcal{R}_{\mathrm{t}}([U] ; \emptyset),\|\phi\|_{\text {sup }}=1\right\}$ is the norm of $\mu_{\emptyset}$ in the space $\mathcal{R}_{\mathrm{t}}^{\prime}([U] ; \emptyset)$.

3.1. Thick delta functions. In order to consider the spaces $\mathcal{R}_{\mathrm{t}}^{\prime}([U] ; F)$ when $F \neq \emptyset$ we need to introduce the idea of thick delta functions.

Definition 3.1. Let $\mathbf{c} \in \bar{U}$. Let $\xi \in \mathcal{M}(\mathbb{S})$. The formula

$$
\left\langle\xi(\mathbf{w}) \delta_{\mathbf{w}}(\mathbf{x}-\mathbf{c}), \phi(\mathbf{x})\right\rangle_{\mathcal{R}_{\mathrm{t}}^{\prime}[U] \times \mathcal{R}_{\mathrm{t}}[U]}=\left\langle\xi(\mathbf{w}), \phi_{\mathbf{c}}(\mathbf{w})\right\rangle_{\mathcal{M}(\mathbb{S}) \times C(\mathbb{S})},
$$

defines an element of $\mathcal{R}_{\mathrm{t}}^{\prime}[U]$ which we call a thick delta function at $\mathbf{x}=\mathbf{c}$, and which we denote as $\xi(\mathbf{w}) \delta_{\mathbf{w}}(\mathbf{x}-\mathbf{c})$ or as $\xi \delta_{*}(\mathbf{x}-\mathbf{c})$.

If $\mathbf{c} \in \bar{U}$ and $\gamma \in \mathbb{R}$, then $\gamma \delta(\mathbf{x}-\mathbf{c})$ is an ordinary delta function at $\mathbf{x}=\mathbf{c}$. In the space $\mathcal{R}^{\prime}[U]$ ordinary delta functions can be expressed as thick deltas, because of $(2.2)$, but in $\mathcal{R}_{\mathrm{t}}^{\prime}[U]$ ordinary deltas and thick deltas are linearly independent.

Remark 3.2. The projection of a thick delta function $\xi(\mathbf{w}) \delta_{\mathbf{w}}(\mathbf{x}-\mathbf{c})$ to the usual space of Radon measures, $(C(\bar{U}))^{\prime}$, is given by a standard delta at $\mathbf{x}=\mathbf{c}$, namely $\gamma \delta(\mathbf{x}-\mathbf{c})$, where $\gamma=\langle\xi(\mathbf{w}), 1\rangle$. This projection might vanish even if the thick delta is not zero: The test functions of $C(\bar{U})$ do not have the ability to detect thick deltas, while the test functions of $\mathcal{R}[U]$ do.

In order to obtain our first main results of this section, we need two short lemmas.

Lemma 3.3. Let $\phi \in \mathcal{R}_{\mathrm{t}}[U]$ and let $g: \mathbb{R} \rightarrow \mathbb{R}$ be continuous with $g(0)=0$. Then $g \circ \phi \in \mathcal{R}_{\mathrm{t}}[U]$.

Proof. Indeed, it is easy to see that $g \circ \phi$ has thick values at each point $\mathbf{x} \in \mathbb{R}^{n}$ given by $(g \circ \phi)_{\mathbf{x}}=g \circ \phi_{\mathbf{x}}$, which is continuous in $\mathbb{S}$.

Lemma 3.4. Let $\phi_{1}, \phi_{2} \in \mathcal{R}_{\mathrm{t}}[U]$, then $\max \left(\phi_{1}, \phi_{2}\right)$ and $\min \left(\phi_{1}, \phi_{2}\right)$ also belong to $\mathcal{R}_{\mathrm{t}}[U]$.

Proof. It follows from the previous lemma, since $\max \left(\phi_{1}, \phi_{2}\right)=\phi_{2}+\left(\phi_{1}-\phi_{2}\right.$ $\left.+\left|\phi_{1}-\phi_{2}\right|\right) / 2$, a composition of addition, substraction, and the absolute value function which is continuous. Similarly, we have that $\min \left(\phi_{1}, \phi_{2}\right)=$ $\phi_{2}+\left(\phi_{1}-\phi_{2}-\left|\phi_{1}-\phi_{2}\right|\right) / 2$. 
We can now characterize the elements of $\mathcal{R}_{\mathrm{t}}^{\prime}[U]$ and of $\mathcal{R}^{\prime}[U]$ with support at a single point. The notion of support for an element $\nu \in \mathcal{R}_{\mathrm{t}}^{\prime}[U]$ is the standard one, namely, $\operatorname{supp} \nu \subset K$, where $K$ is a closed subset of $\bar{U}$ if $\langle\nu, \phi\rangle=0$ for $\phi \in \mathcal{R}_{\mathrm{t}}^{\prime}[U]$ with $\phi(\mathbf{x})=0$ for all $\mathbf{x} \in K$.

Let us start with the space $\mathcal{R}^{\prime}[U]$.

Proposition 3.5. Let $\mu \in \mathcal{R}^{\prime}[U]$ with $\operatorname{supp} \mu=\{\mathbf{c}\}$. Then there exists $\xi \in \mathcal{M}(\mathbb{S})$ such that

$$
\mu(\mathbf{x})=\xi(\mathbf{w}) \delta_{\mathbf{w}}(\mathbf{x}-\mathbf{c}) .
$$

Proof. Let us define a functional

$$
\begin{aligned}
\xi_{0}: E & \rightarrow \mathbb{R} \\
\phi_{c} & \longmapsto\langle\mu, \phi\rangle
\end{aligned}
$$

where $E=\left\{\phi_{c}: \phi \in \mathcal{R}_{\mathrm{t}}[U]\right\}$, a linear subspace of $C(\mathbb{S})$.

Observe first that $\xi_{0}$ is well-defined. Namely, if $\phi_{c}=\psi_{c}$ for different $\phi$ and $\psi$, then $\left\langle\xi_{0}, \phi_{c}\right\rangle=\langle\mu, \phi\rangle=\langle\mu, \psi\rangle=\left\langle\xi_{0}, \psi_{c}\right\rangle$ since $\phi-\psi$ vanishes at $\mathbf{c}$ and thus $\langle\mu, \phi-\psi\rangle=0$.

Next we shall show that there exists a $\xi \in \mathcal{M}(\mathbb{S})$ such that $\left.\xi\right|_{E}=\xi_{0}$. This gives $\mu(\mathbf{x})=\xi(\mathbf{w}) \delta_{\mathbf{w}}(\mathbf{x}-\mathbf{c})$, as required. The existence of $\xi$ would follow from the Hahn-Banach theorem if we show that $\xi_{0}$ is a continuous linear functional. The linearity is clear, so we only need to prove continuity, or equivalently, boundedness. Let $\phi \in \mathcal{R}[U]$, and let $\phi_{\mathbf{c}}=\alpha \in E$. For any $\varepsilon>0$, the function $\zeta$ defined by

$$
\zeta(\mathbf{x})=\left\{\begin{array}{cl}
\phi(\mathbf{x}), & \text { if }|\phi(\mathbf{x})| \leq\|\alpha\|_{\text {sup }}+\varepsilon \\
\|\alpha\|_{\text {sup }}+\varepsilon, & \text { if } \phi(\mathbf{x}) \geq\|\alpha\|_{\text {sup }}+\varepsilon \\
-\left(\|\alpha\|_{\text {sup }}+\varepsilon\right), & \text { if } \phi(\mathbf{x}) \leq-\left(\|\alpha\|_{\text {sup }}+\varepsilon\right)
\end{array}\right.
$$

belongs to $\mathcal{R}_{\mathrm{t}}[U]$ because of the lemma 3.4. Let $\psi=N(\zeta)$, so that $\psi \in \mathcal{R}[U]$ and $\psi_{\mathbf{c}}=\zeta_{\mathbf{c}}=\phi_{\mathbf{c}}=\alpha$. Hence, $\left|\left\langle\xi_{0}, \alpha\right\rangle\right| \leq\|\mu\|\|\psi\|_{\text {sup }} \leq\|\mu\|\left(\|\alpha\|_{\text {sup }}+\varepsilon\right)$. Since $\varepsilon$ is arbitrary,

$$
\left|\left\langle\xi_{0}, \alpha\right\rangle\right| \leq\|\mu\|\|\alpha\|_{\text {sup }} .
$$

Therefore, there exists $\xi \in \mathcal{M}(\mathbb{S})$, with $\|\xi\|=\|\mu\|$, that extends $\xi_{0}$ to all of $C(\mathbb{S})$.

The corresponding result for $\mathcal{R}_{\mathrm{t}}^{\prime}[U]$ is the following.

Proposition 3.6. Let $\nu \in \mathcal{R}_{\mathrm{t}}^{\prime}[U]$ with $\operatorname{supp} \nu=\{\mathbf{c}\}$. Then there exists $\xi \in \mathcal{M}(\mathbb{S})$ and $\gamma \in \mathbb{R}$ such that

$$
\nu(\mathbf{x})=\xi(\mathbf{w}) \delta_{\mathbf{w}}(\mathbf{x}-\mathbf{c})+\gamma \delta(\mathbf{x}-\mathbf{c}) .
$$


Proof. Let $\nu \in \mathcal{R}_{\mathrm{t}}^{\prime}[U]$ with $\operatorname{supp} \nu=\{\mathbf{c}\}$. Since $\mathcal{R}_{\mathrm{t}}[U]=\mathcal{R}[U] \oplus \mathcal{R}_{\mathrm{n}}[U], \nu$ can be decomposed as $\mu_{1}+\mu_{2}$, where $\mu_{1} \in \mathcal{R}^{\prime}[U]$ and $\mu_{2} \in \mathcal{R}_{\mathrm{n}}^{\prime}[U]$. Clearly both $\mu_{1}$ and $\mu_{2}$ have support contained in $\{\mathbf{c}\}$. But $\phi \in \mathcal{R}_{\mathrm{n}}[U]$ if and only if there exists a sequence of different points of $\bar{U},\left\{\mathbf{x}_{n}\right\}_{n=1}^{\infty}$, such that $\phi(\mathbf{x})=0$ if $\mathbf{x} \neq \mathbf{x}_{n}$ for all $n$, and $\lim _{n \rightarrow \infty} \phi\left(\mathbf{x}_{n}\right)=0$. Therefore $\mu_{2}=\gamma \delta(\mathbf{x}-\mathbf{c})$ for some real number $\gamma$. Formula (3.9) now follows from Proposition 3.5 .

In fact, if the support point $\mathbf{c}$ is not on the boundary $\partial U, \xi$ could be uniquely found in the following way.

Proposition 3.7. Let $\mu \in \mathcal{R}^{\prime}[U]$ with $\operatorname{supp} \mu=\{\mathbf{c}\} \subset U$. Then there exists a unique $\xi \in \mathcal{M}(\mathbb{S})$ such that

$$
\mu(\mathbf{x})=\xi(\mathbf{w}) \delta_{\mathbf{w}}(\mathbf{x}-\mathbf{c}) .
$$

Uniqueness of $\xi$ and $\gamma$ in (3.9) also holds if $\nu \in \mathcal{R}_{\mathrm{t}}^{\prime}[U]$ with $\operatorname{supp} \nu=\{\mathbf{c}\} \subset$ $U$.

Proof. Let $\rho \in \mathcal{R}[U]$ be a continuous function in $\mathbb{R}^{n}$ that satisfies $\rho(\mathbf{c})=1$ and $0 \leq \rho(\mathbf{x}) \leq 1$ in all $\mathbb{R}^{n}$. If $\alpha \in C(\mathbb{S})$, let $A(\alpha) \in \mathcal{R}[U]$ be the function given by

$$
A(\alpha)(\mathbf{x})= \begin{cases}\rho(\mathbf{x}) \alpha\left(\frac{\mathbf{x}-\mathbf{c}}{|\mathbf{x}-\mathbf{c}|}\right), & \mathbf{x} \neq \mathbf{c}, \\ \frac{1}{c_{n}} \int_{\mathbb{S}} \alpha(\mathbf{w}) \mathrm{d} \sigma(\mathbf{w}), & \mathbf{x}=\mathbf{c} .\end{cases}
$$

Then $A: C(\mathbb{S}) \rightarrow \mathcal{R}[U]$ is a continuous linear operator of norm 1 . Hence the linear functional $\xi$ defined by $\langle\xi, \alpha\rangle=\langle\mu, A(\alpha)\rangle$ is continuous, $\xi \in \mathcal{M}(\mathbb{S})$.

If $\phi \in \mathcal{R}[U]$ we can write $\phi=A\left(\phi_{\mathbf{c}}\right)+\psi$, where $\psi_{\mathbf{c}}=0$, and it follows that

$$
\langle\mu, \phi\rangle=\left\langle\mu, A\left(\phi_{\mathbf{c}}\right)\right\rangle+\langle\mu, \psi\rangle=\left\langle\mu, A\left(\phi_{\mathbf{c}}\right)\right\rangle=\left\langle\xi, \phi_{\mathbf{c}}\right\rangle,
$$

so that $\mu(\mathbf{x})=\xi(\mathbf{w}) \delta_{\mathbf{w}}(\mathbf{x}-\mathbf{c})$.

In the case of formula (3.9) in $\mathcal{R}_{\mathrm{t}}^{\prime}[U]$, it is clear that $\gamma$ is unique, whether c belongs to the interior or the boundary of $U$, while the uniqueness of $\xi$ follows from the first part of the proof when $\mathbf{c} \in U$.

Similarly, if $\nu \in \mathcal{R}_{\mathrm{t}}^{\prime}([U] ; F)$, where $F=G \cup\{\mathbf{c}\}$, is a finite set and the restriction of $\nu$ to $\mathcal{R}_{\mathrm{t}}([U] ; G)$ vanishes, then $\nu$ could be expressed as:

$$
\begin{aligned}
\nu(\mathbf{x}) & =\mu_{1}+\mu_{2} \\
& =\xi(\mathbf{w}) \delta_{\mathbf{w}}(\mathbf{x}-\mathbf{c})+\gamma \delta(\mathbf{x}-\mathbf{c}),
\end{aligned}
$$

where $\mu_{1} \in \mathcal{R}^{\prime}[U]$ is a thick delta function and $\mu_{2}=\gamma \delta(\mathbf{x}-\mathbf{c}) \in \mathcal{R}_{\mathrm{n}}^{\prime}[U]$ is an ordinary delta function.

If we now start from (3.1) and (3.2), and employ an inductive argument, we obtain the ensuing formula. 
Proposition 3.8. Let $\nu \in \mathcal{R}_{\mathrm{t}}^{\prime}([U] ; F)$, where $F$ is a finite set of points, then

$$
\nu=\mu_{\mathrm{cont}}+\sum_{n=1}^{\infty} \gamma_{n} \delta\left(\mathbf{x}-\mathbf{x}_{n}\right)+\sum_{\mathbf{c} \in F} \xi_{\mathbf{c}} \delta_{*}(\mathbf{x}-\mathbf{c}),
$$

where $\xi_{c} \in \mathcal{M}(\mathbb{S})$ so that $\xi_{c} \delta_{*}(\mathbf{x}-\mathbf{c})$ is a thick delta at $\mathbf{x}=\mathbf{c}$.

A similar formula holds in $\mathcal{R}^{\prime}[U]$.

Proposition 3.9. Let $\mu \in \mathcal{R}^{\prime}([U] ; F)$, where $F$ is a finite set of points, then

$$
\mu=\mu_{\text {cont }}+\sum_{\substack{n=1 \\ \mathbf{x}_{n} \notin F}}^{\infty} \gamma_{n} \delta\left(\mathbf{x}-\mathbf{x}_{n}\right)+\sum_{\mathbf{c} \in F} \xi_{\mathbf{c}} \delta_{*}(\mathbf{x}-\mathbf{c}),
$$

where $\xi_{c} \in \mathcal{M}(\mathbb{S})$ so that $\xi_{c} \delta_{*}(\mathbf{x}-\mathbf{c})$ is a thick delta at $\mathbf{x}=\mathbf{c}$.

Notice that if $\mathbf{c} \in F$ but $\mathbf{c} \neq \mathbf{x}_{n}$ for all $n$, then $\left\langle\xi_{\mathbf{c}}, 1\right\rangle=0$, while if $\mathbf{c}=\mathbf{x}_{n}$ for some $n$, then $\left\langle\xi_{\mathbf{c}}, 1\right\rangle=\gamma_{n}$.

We are almost ready to give a characterization of the dual spaces $\mathcal{R}_{\mathrm{t}}^{\prime}[U]$ and $\mathcal{R}^{\prime}[U]$. We need to use the fact that the family of restrictions $\left\{\mu_{F}\right\}_{F}$ finite of an element $\mu$ of $\mathcal{R}_{\mathrm{t}}^{\prime}[U]$ (or $\mathcal{R}^{\prime}[U]$ ) to the spaces $\mathcal{R}_{\mathrm{t}}^{\prime}([U] ; F)$ (or $\mathcal{R}^{\prime}([U] ; F)$ ) determines $\mu$ uniquely. This will follow from the density result of the next section.

3.2. A density result. Our next aim is to show that the union of the sets $\mathcal{R}_{\mathrm{t}}([U] ; F)$ for $F$ finite is dense in $\mathcal{R}_{\mathrm{t}}[U]$, and a corresponding result in $\mathcal{R}[U]$.

If $f$ is a function defined in a set $E$ and with real values we shall use the notation

$$
\operatorname{Var}(f ; E)=\sup \{|f(x)-f(y)|: x, y \in E\},
$$

for the variation of $f$ over the set $E$. If $f$ is defined in an open set of $\mathbb{R}^{n}$ it will also be convenient to introduce the variation functions

$$
\begin{aligned}
& v_{f}(\mathbf{x})=\lim _{\varepsilon \rightarrow 0} \operatorname{Var}\left(f ; B_{\mathbf{x}}(\varepsilon)\right), \\
& v_{f}^{*}(\mathbf{x})=\lim _{\varepsilon \rightarrow 0} \operatorname{Var}\left(f ; B_{\mathbf{x}}^{*}(\varepsilon)\right),
\end{aligned}
$$

where $B_{\mathbf{x}}(\varepsilon)$ is the ball of center $\mathbf{x}$ and radius $\varepsilon$ and $B_{\mathbf{x}}^{*}(\varepsilon)$ is the corresponding punctured ball, $B_{\mathbf{x}}^{*}(\varepsilon)=B_{\mathbf{x}}(\varepsilon) \backslash\{\mathbf{x}\}$.

Notice that $0 \leq v_{f}^{*}(\mathbf{x}) \leq v_{f}(\mathbf{x})$; also observe that if $\phi \in \mathcal{R}_{\mathrm{t}}[U]$, then

$$
v_{\phi}^{*}(\mathbf{x})=\operatorname{Var}\left(\phi_{\mathbf{x}} ; \mathbb{S}\right) .
$$

Interestingly, if $\phi \in \mathcal{R}_{\mathrm{t}}[U]$ then the variation function $v_{\phi}$ actually belongs to $\mathcal{R}_{\mathrm{n}}[U]$. 
Proposition 3.10. If $\phi \in \mathcal{R}_{\mathrm{t}}[U]$, then for all $\mathbf{x} \in \mathbb{R}^{n}$,

$$
\lim _{\mathbf{z} \rightarrow \mathbf{x}} v_{\phi}(\mathbf{z})=0 \text {. }
$$

Proof. Let $\mathbf{x}$ be fixed. Let $\varepsilon>0$. Then there exists $r_{0}>0$ such that

$$
\left|\phi_{\mathbf{x}}(\mathbf{w})-\phi(\mathbf{x}+r \mathbf{w})\right|<\frac{\varepsilon}{3}, \quad 0<r<r_{0}, \quad \forall \mathbf{w} \in \mathbb{S} .
$$

But $\phi_{\mathbf{x}}$ is continuous, so there exists $\delta>0$ such that if $\left|\mathbf{w}_{1}-\mathbf{w}_{2}\right|<\delta$, then $\left|\phi_{\mathbf{x}}\left(\mathbf{w}_{1}\right)-\phi_{\mathbf{x}}\left(\mathbf{w}_{2}\right)\right|<\varepsilon / 3$.

If $\mathbf{z} \in B_{\mathbf{x}}^{*}\left(r_{0}\right)$, and $\eta>0$ is small, then for any $\mathbf{y}_{1}, \mathbf{y}_{2} \in B_{\mathbf{z}}(\eta)$, we can write

$$
\mathbf{y}_{1}=\mathbf{x}+r_{1} \mathbf{w}_{1}, \quad \mathbf{y}_{2}=\mathbf{x}+r_{2} \mathbf{w}_{2},
$$

with $r_{j}<r_{0}$, and $\left|\mathbf{w}_{1}-\mathbf{w}_{2}\right|<\delta$, so that we have

$$
\begin{aligned}
& \left|\phi\left(\mathbf{y}_{1}\right)-\phi\left(\mathbf{y}_{2}\right)\right| \\
& \quad \leq\left|\phi\left(\mathbf{y}_{1}\right)-\phi_{\mathbf{x}}\left(\mathbf{w}_{1}\right)\right|+\left|\phi\left(\mathbf{w}_{1}\right)-\phi_{x}\left(\mathbf{w}_{2}\right)\right|+\left|\phi\left(\mathbf{w}_{2}\right)-\phi_{\mathbf{x}}\left(\mathbf{y}_{2}\right)\right|<\varepsilon .
\end{aligned}
$$

Hence $v_{\phi}(\mathbf{z}) \leq \varepsilon$.

Employing (3.19) it follows that if $\phi \in \mathcal{R}_{\mathrm{t}}[U]$ then for any $\varepsilon>0$ the set

$$
E_{\varepsilon}=\left\{\mathbf{x}: v_{\phi}(\mathbf{x}) \geq \varepsilon\right\}
$$

is finite. This allows us to obtain the following decomposition of regulated functions of $\mathcal{R}_{\mathrm{t}}[U]$.

Proposition 3.11. Let $\phi \in \mathcal{R}_{\mathrm{t}}[U]$ and let $\varepsilon>0$. Then there exist functions $\phi_{\varepsilon} \in \mathcal{R}_{\mathrm{t}}\left([U] ; E_{\varepsilon}\right)$ and $\psi \in \mathcal{R}_{\mathrm{t}}[U]$ with $v_{\psi}(\mathbf{x})<\varepsilon$ for all $\mathbf{x}$ such that

$$
\phi=\phi_{\varepsilon}+\psi \text {. }
$$

Proof. We shall show that if $\mathbf{c} \in U$ then we can find a function $\varphi^{\mathbf{c}} \in$ $\mathcal{R}_{\mathrm{t}}([U] ;\{\mathbf{c}\})$ such that $\phi_{\mathbf{c}}=\left(\varphi^{\mathbf{c}}\right)_{\mathbf{c}}$, while if $\mathbf{c} \in \partial U$ and $\eta>0$ then we can find a function $\zeta^{\mathbf{c}} \in \mathcal{R}_{\mathrm{t}}([U] ;\{\mathbf{c}\})$ such that $v_{\phi-\zeta^{\mathbf{c}}}(\mathbf{c}) \leq \eta$. Then one can take $\eta<\varepsilon$ and

$$
\phi_{\varepsilon}=\sum_{\mathbf{c} \in E_{\varepsilon} \cap U} \varphi^{\mathbf{c}}+\sum_{\mathbf{c} \in E_{\varepsilon} \cap \partial U} \zeta^{\mathbf{c}} .
$$

First consider the simpler case when $\mathbf{c} \in U$. Let $\delta>0$ such that the closed ball $\overline{B_{\mathbf{c}}(\delta)} \subset U$ and let $\rho \in C(\mathbb{R})$ be such that $\rho(t)=0$ for $t \geq \delta$ and $\rho(t)=1$ for $t \leq \delta / 2$. Then the function $\varphi^{\mathbf{c}}$ defined as

$$
\varphi^{\mathbf{c}}(\mathbf{c}+t \mathbf{w})= \begin{cases}\rho(t) \phi_{\mathbf{c}}(\mathbf{w}), & t>0, \\ \phi(\mathbf{c}), & t=0,\end{cases}
$$

belongs to $\mathcal{R}_{\mathrm{t}}([U] ;\{\mathbf{c}\})$ and $\phi_{\mathbf{c}}=\left(\varphi^{\mathbf{c}}\right)_{\mathbf{c}}$. 
Suppose now that $\mathbf{c} \in \partial U$ and $\eta>0$. There exists $\delta>0$ such that $\left|\phi_{\mathbf{c}}(\mathbf{w})-\phi(\mathbf{c}+t \mathbf{w})\right|<\eta / 2$ for $0<t \leq \delta$ and for all $\mathbf{w} \in \mathbb{S}$. Let us also consider the following closed subsets of $\mathbb{S}, H=\left\{\mathbf{w} \in \mathbb{S}:\left|\phi_{\mathbf{c}}(\mathbf{w})\right| \leq \eta / 2\right\}$ and $K=\left\{\mathbf{w} \in \mathbb{S}:\left|\phi_{\mathbf{c}}(\mathbf{w})\right| \geq \eta\right\}$. We can find a function $\chi \in C(\mathbb{S})$ with $0 \leq \chi \leq 1$ such that $\chi(\mathbf{w})=0$ for $\mathbf{w} \in H$ and $\chi(\mathbf{w})=1$ for $\mathbf{w} \in K$. Let $\alpha=\chi \phi_{\mathbf{c}} \in C(\mathbb{S})$. Observe that $\left\|\phi_{\mathbf{c}}-\alpha\right\|_{\text {sup }} \leq \eta$.

Let $\rho \in C(\mathbb{R})$ be such that $\rho(t)=0$ for $t \geq \delta$ and $\rho(t)=1$ for $t \leq \delta / 2$. Define the function

$$
\zeta^{\mathbf{c}}(\mathbf{c}+t \mathbf{w})= \begin{cases}\rho(t) \alpha(\mathbf{w}), & t>0, \\ \phi(\mathbf{c}), & t=0 .\end{cases}
$$

Then $\zeta^{\mathbf{c}}$ is a $\mathcal{B}_{s}(\mathbb{S})$-regulated function, continuous in $\mathbb{R}^{n} \backslash\{\mathbf{c}\}$, and with $\left(\zeta^{\mathbf{c}}\right)_{\mathbf{c}}=\alpha$. If we show that $\zeta^{\mathbf{c}} \in \mathcal{R}_{\mathrm{t}}[U]$ the proof would be complete, and in order to show that $\zeta^{\mathbf{c}} \in \mathcal{R}_{\mathrm{t}}[U]$ all we need to show is that $\zeta^{\mathbf{c}}(\mathbf{x})=0$ if $\mathbf{x} \in \mathbb{R}^{n} \backslash \bar{U}$. But $\zeta^{\mathbf{c}}(\mathbf{x})=0$ if $|\mathbf{x}-\mathbf{c}| \geq \delta$, while if $\mathbf{x} \in \mathbb{R}^{n} \backslash \bar{U}$ and $|\mathbf{x}-\mathbf{c}|<\delta$, writing $\mathbf{x}=\mathbf{c}+t \mathbf{w}$ we have that $\mathbf{w} \in H$, since $\phi(\mathbf{x})=0$, and $\left|\phi_{\mathbf{c}}(\mathbf{w})-\phi(\mathbf{c}+t \mathbf{w})\right|<\eta / 2$; thus $\alpha(\mathbf{w})=0$ and, consequently, $\zeta^{\mathbf{c}}(\mathbf{x})=0$.

We shall also need the following approximation result.

Proposition 3.12. Let $\phi \in \mathcal{R}_{\mathrm{t}}[U]$ with $v_{\phi}(\mathbf{x})<\varepsilon$ for all $\mathbf{x} \in \mathbb{R}^{n}$. Then there exists a continuous function $f \in \mathcal{R}_{\mathrm{t}}[U]$ such that $|\phi(\mathbf{x})-f(\mathbf{x})|<2 \varepsilon$ for all $\mathbf{x} \in \mathbb{R}^{n}$.

Proof. Since $v_{\phi}(\mathbf{x})<\varepsilon$ for all $\mathbf{x} \in \bar{U}$, and $\bar{U}$ is compact, we have that $\max _{\mathbf{x} \in \bar{U}} v_{\phi}(\mathbf{x})<\varepsilon$. Furthermore, if $\max _{\mathbf{x} \in \bar{U}} v_{\phi}(\mathbf{x})<\eta<\varepsilon$ we can find $\delta>0$ such that

$$
\operatorname{Var}\left(\phi ; B_{\mathbf{x}}(\delta)\right)<\eta,
$$

for all $\mathbf{x} \in \mathbb{R}^{n}$.

Let $\rho \in C\left(\mathbb{R}^{n}\right)$, be a continuous function on $\mathbb{R}^{n}$ such that $\rho(\mathbf{x})=0$ when $|\mathbf{x}| \geq \delta, \rho(\mathbf{x}) \geq 0$ when $|\mathbf{x}| \leq \delta$, and

$$
\int_{\mathbb{R}^{n}} \rho(\mathbf{x}) \mathrm{d} \mathbf{x}=1 .
$$

Then we can approximate any $\psi \in \mathcal{R}_{\mathrm{t}}[U]$ with the convolution $g=\psi * \rho$, which is a continuous function. Indeed, we have,

$$
\begin{aligned}
|g(\mathbf{x})-\psi(\mathbf{x})| & =\left|\int_{\mathbb{R}^{n}} \psi(\mathbf{x}-\mathbf{z}) \rho(\mathbf{z}) \mathrm{d} \mathbf{z}-\psi(\mathbf{x})\right| \\
& =\left|\int_{\mathbb{R}^{n}} \psi(\mathbf{x}-\mathbf{z}) \rho(\mathbf{z}) \mathrm{d} \mathbf{z}-\int_{\mathbb{R}^{n}} \psi(\mathbf{x}) \rho(\mathbf{z}) \mathrm{d} \mathbf{z}\right| \\
& \leq \int_{|\mathbf{z}| \leq \delta}|\psi(\mathbf{x}-\mathbf{z})-\psi(\mathbf{x})| \rho(\mathbf{z}) \mathrm{d} \mathbf{z}
\end{aligned}
$$




$$
\leq \eta \int_{\mathbb{R}^{n}} \rho(\mathbf{z}) \mathrm{d} \mathbf{z}=\eta
$$

While $g$ has compact support, this support may or may not be contained in $\bar{U}$. Therefore we proceed as follows. Let $\gamma>0$ be such that $\eta+\gamma<\varepsilon$. Let $K=\left\{\mathbf{x} \in \mathbb{R}^{n}:|g(\mathbf{x})| \geq \eta+\gamma\right\}$; we can find a continuous function $h \in$ $C\left(\mathbb{R}^{n}\right)$ with $0 \leq h \leq 1$, such that $h(\mathbf{x})=1$ if $\mathbf{x} \in K$ and $h(\mathbf{x})=0$ if $\mathbf{x} \in \mathbb{R}^{n} \backslash U$, since $g(\mathbf{x}) \leq \eta$ if $\mathbf{x} \in \mathbb{R}^{n} \backslash U$. Put $f=h g$. Then $f \in \mathcal{R}_{\mathrm{t}}([U] ; \emptyset)$ and if $\mathbf{x} \in K,|f(\mathbf{x})-\phi(\mathbf{x})|=|g(\mathbf{x})-\phi(\mathbf{x})| \leq \eta<2 \varepsilon$, while if $\mathbf{x} \notin K$,

$$
|f(\mathbf{x})-\phi(\mathbf{x})| \leq|f(\mathbf{x})-g(\mathbf{x})|+|g(\mathbf{x})-\phi(\mathbf{x})| \leq 2 \eta+\gamma<2 \varepsilon,
$$

so that $\|f-\phi\|_{\text {sup }}<2 \varepsilon$.

We can now prove the density of the union of the spaces $\mathcal{R}_{\mathrm{t}}([U] ; F)$ in $\mathcal{R}_{\mathrm{t}}[U]$. This means that if $\phi \in \mathcal{R}_{\mathrm{t}}[U]$, then we can find a sequence of finite sets $\left\{F_{m}\right\}_{m=1}^{\infty}$ and regulated functions $\phi_{m} \in \mathcal{R}_{\mathrm{t}}\left([U] ; F_{m}\right)$, such that $\phi_{m} \rightarrow \phi$ uniformly in $\mathbb{R}^{n}$.

Theorem 3.13. If $U$ is an open bounded set of $\mathbb{R}^{n}$ then

$$
\bigcup_{F \text { finite }} \mathcal{R}_{\mathrm{t}}([U] ; F)=\mathcal{R}_{\mathrm{t}}[U] .
$$

Proof. Indeed, if $\phi \in \mathcal{R}_{\mathrm{t}}[U]$ and $\varepsilon>0$, Proposition 3.11 allows us to write $\phi=\zeta+\psi$, where $\zeta \in \mathcal{R}_{\mathrm{t}}([U] ; F), F$ is finite, and $\psi \in \mathcal{R}_{\mathrm{t}}[U]$ with $v_{\psi}(\mathbf{x})<$ $\varepsilon / 2$ for all $\mathbf{x}$. On the other hand, Proposition 3.12 gives the existence of $f \in \mathcal{R}_{\mathrm{t}}([U] ; \emptyset)$ such that $\|\psi-f\|_{\text {sup }}<\varepsilon$. Then $\phi_{F}=\zeta+f$ belongs to $\mathcal{R}_{\mathrm{t}}([U] ; F)$ and $\left\|\phi-\phi_{F}\right\|_{\text {sup }}<\varepsilon$.

We also have a density result in $\mathcal{R}[U]$.

Theorem 3.14. If $U$ is an open bounded set of $\mathbb{R}^{n}$ then

$$
\bigcup_{F \text { finite }} \mathcal{R}([U] ; F)=\mathcal{R}[U] \text {. }
$$

Proof. If $\phi \in \mathcal{R}[U]$ and $\varepsilon>0$, we can find a finite set $F$ and $\phi_{F} \in$ $\mathcal{R}_{\mathrm{t}}([U] ; F)$ such that $\left\|\phi-\phi_{F}\right\|_{\text {sup }}<\varepsilon$. Then $N\left(\phi_{F}\right) \in \mathcal{R}([U] ; F)$ and $\left\|\phi-N\left(\phi_{F}\right)\right\|_{\text {sup }}=\left\|N\left(\phi-\phi_{F}\right)\right\|_{\text {sup }} \leq\left\|\phi-\phi_{F}\right\|_{\text {sup }}<\varepsilon$.

3.3. Description of the dual spaces $\mathcal{R}_{\mathrm{t}}^{\prime}[U]$ and $\mathcal{R}^{\prime}[U]$. Each $\nu \in \mathcal{R}_{\mathrm{t}}^{\prime}[U]$ has associated a family of elements $\left\{\mu_{F}\right\}_{F}$ finite of the duals of the spaces $\mathcal{R}_{\mathrm{t}}([U] ; F)$, for $F$ finite, obtained by restriction. Our density results guarantee that this family determines $\nu$ uniquely. A similar situation holds in $\mathcal{R}^{\prime}[U]$. Since we know the form of the elements of $\mathcal{R}_{\mathrm{t}}^{\prime}([U] ; F)$ and $\mathcal{R}^{\prime}([U] ; F)$, we immediately obtain the form of the elements of the spaces $\mathcal{R}_{\mathrm{t}}^{\prime}[U]$ and $\mathcal{R}^{\prime}[U]$ as signed measures with a sum of thick deltas. 
Theorem 3.15. Let $U$ be a bounded open set of $\mathbb{R}^{n}$. Let $\mu \in \mathcal{R}^{\prime}[U]$. Then there exists a countable set $\mathfrak{S} \subset \bar{U}$, signed measures $\xi_{\mathbf{c}} \in \mathcal{M}(\mathbb{S})$ for $\mathbf{c} \in \mathfrak{S}$, and a continuous Radon measure $\mu_{\text {cont }}$ of finite mass in $U$ such that

$$
\mu(\mathbf{x})=\mu_{\text {cont }}(\mathbf{x})+\sum_{\mathbf{c} \in \mathfrak{S}} \xi_{\mathbf{c}} \delta_{*}(\mathbf{x}-\mathbf{c}) .
$$

The norm of $\mu$ in the space $\mathcal{R}^{\prime}[U]$, is given as

$$
\|\mu\|_{\mathcal{R}^{\prime}[U]}=\int_{U} \mathrm{~d}\left|\mu_{\text {cont }}\right|+\sum_{\mathbf{c} \in \mathfrak{S}}\left\|\xi_{\mathbf{c}}\right\|_{\mathcal{M}(\mathbb{S})} .
$$

Similarly, we have the ensuing characterization.

Theorem 3.16. Let $U$ be a bounded open set of $\mathbb{R}^{n}$. Let $\nu \in \mathcal{R}_{\mathrm{t}}^{\prime}[U]$. Then there exists a countable set $\mathfrak{S} \subset \bar{U}$, signed measures $\xi_{\mathbf{c}} \in \mathcal{M}(\mathbb{S})$ and numbers $\gamma_{\mathbf{c}}$ for $\mathbf{c} \in \mathfrak{S}$, and a continuous Radon measure $\mu_{\mathrm{cont}}$ of finite mass in $U$ such that

$$
\nu(\mathbf{x})=\mu_{\text {cont }}(\mathbf{x})+\sum_{\mathbf{c} \in \mathfrak{S}}\left(\xi_{\mathbf{c}} \delta_{*}(\mathbf{x}-\mathbf{c})+\gamma_{\mathbf{c}} \delta(\mathbf{x}-\mathbf{c})\right) .
$$

The norm of $\nu$ in the space $\mathcal{R}_{\mathrm{t}}^{\prime}[U]$, is given as

$$
\|\nu\|_{\mathcal{R}_{\mathrm{t}}^{\prime}[U]}=\int_{U} \mathrm{~d}\left|\mu_{\text {cont }}\right|+\sum_{\mathbf{c} \in \mathfrak{S}}\left(\left\|\xi_{\mathbf{c}}\right\|_{\mathcal{M}(\mathbb{S})}+\left|\gamma_{\mathbf{c}}\right|\right) .
$$

\section{Spaces OVER General OPEN SETS}

We shall now consider the dual spaces of several spaces of regulated functions defined on a general open set $\Omega \subset \mathbb{R}^{n}$.

The space $C_{0}\left(\mathbb{R}^{n}\right)$ consists of the continuous functions that vanish at infinity, a Banach space with the supremum norm. Similarly we can define the spaces $\mathcal{R}_{0}\left(\mathbb{R}^{n}\right)$, the space of normalized $\mathcal{B}_{s}(\mathbb{S})$-regulated functions such that the thick limits $\phi_{\mathbf{x}_{0}}$ are continuous functions in $\mathbb{S}$ for each $\mathbf{x}_{0} \in \mathbb{R}^{n}$ and that have limit zero at infinity, and $\mathcal{R}_{\mathrm{t}, 0}\left(\mathbb{R}^{n}\right)$, the space of $\mathcal{B}_{s}(\mathbb{S})$-regulated functions such that the thick limits $\phi_{\mathbf{x}_{0}}$ are continuous functions in $\mathbb{S}$ for each $\mathbf{x}_{0} \in \mathbb{R}^{n}$ and that have limit zero at infinity; both are Banach spaces with the supremum norm. The dual space $\left(C_{0}\left(\mathbb{R}^{n}\right)\right)^{\prime}$ is the space of signed measures of total finite variation in $\mathbb{R}^{n}$, and we obtain similar results for the corresponding spaces of regulated functions.

Theorem 4.1. Let $\mu \in \mathcal{R}_{0}^{\prime}\left(\mathbb{R}^{n}\right)$. Then there exists a continuous Radon measure in $\mathbb{R}^{n}, \mu_{\text {cont }}$, a countable subset $\mathfrak{S} \subset \mathbb{R}^{n}$, and signed measures $\xi_{\mathbf{c}} \in \mathcal{M}(\mathbb{S})$ for $\mathbf{c} \in \mathfrak{S}$ such that

$$
\mu(\mathbf{x})=\mu_{\text {cont }}(\mathbf{x})+\sum_{\mathbf{c} \in \mathfrak{S}} \xi_{\mathbf{c}} \delta_{*}(\mathbf{x}-\mathbf{c}) .
$$


Furthermore, the norm of $\mu$ in the space $\mathcal{R}_{0}^{\prime}\left(\mathbb{R}^{n}\right)$ is

$$
\|\mu\|=\int_{\mathbb{R}^{n}} \mathrm{~d}\left|\mu_{\text {cont }}\right|+\sum_{\mathbf{c} \in \mathfrak{S}}\left\|\xi_{\mathbf{c}}\right\|_{\mathcal{M}(\mathbb{S})} .
$$

Let $\nu \in \mathcal{R}_{\mathrm{t}, 0}^{\prime}\left(\mathbb{R}^{n}\right)$. Then there exists a continuous Radon measure in $\mathbb{R}^{n}$, $\mu_{\text {cont }}$, a countable subset $\mathfrak{S} \subset \mathbb{R}^{n}$, signed measures $\xi_{\mathbf{c}} \in \mathcal{M}(\mathbb{S})$ and constants $\gamma_{\mathbf{c}} \in \mathbb{R}$ for $\mathbf{c} \in \mathfrak{S}$ such that

$$
\nu(\mathbf{x})=\mu_{\text {cont }}(\mathbf{x})+\sum_{\mathbf{c} \in \mathfrak{S}}\left(\xi_{\mathbf{c}} \delta_{*}(\mathbf{x}-\mathbf{c})+\gamma_{\mathbf{c}} \delta(\mathbf{x}-\mathbf{c})\right)
$$

Moreover, the norm of $\nu$ in the space $\mathcal{R}_{\mathrm{t}, 0}^{\prime}\left(\mathbb{R}^{n}\right)$ is

$$
\|\nu\|=\int_{\mathbb{R}^{n}} \mathrm{~d}\left|\mu_{\text {cont }}\right|+\sum_{\mathbf{c} \in \mathfrak{S}}\left(\left\|\xi_{\mathbf{c}}\right\|_{\mathcal{M}(\mathbb{S})}+\left|\gamma_{\mathbf{c}}\right|\right) .
$$

The spaces $\mathcal{R}(\Omega)$ and $\mathcal{R}_{\mathrm{t}}(\Omega)$ are the analogous of $C(\Omega)$. A function $\phi$ belongs to $\mathcal{R}_{\mathrm{t}}(\Omega)$ if it is a $\mathcal{B}_{s}(\mathbb{S})$-regulated functions defined in all $\Omega$ such that the thick limits $\phi_{\mathbf{x}_{0}}$ are continuous functions in $\mathbb{S}$ for each $\mathbf{x}_{0} \in \Omega$, without any restriction on its behavior at the boundary; it belongs to $\mathcal{R}(\Omega)$ if it is a normalized function in $\mathcal{R}_{\mathrm{t}}(\Omega)$. The spaces $\mathcal{R}(\Omega)$ and $\mathcal{R}_{\mathrm{t}}(\Omega)$ are, like $C(\Omega)$, topological vector spaces, but not Banach spaces: Convergence means uniform convergence over each compact subset of $\Omega$. The dual space $(C(\Omega))^{\prime}$ is the space of signed measures with compact support in $\Omega$, and thus the dual spaces of $\mathcal{R}(\Omega)$ and $\mathcal{R}_{\mathrm{t}}(\Omega)$ are formed by signed measures with thick deltas and compact support.

Theorem 4.2. Let $\mu \in \mathcal{R}^{\prime}(\Omega)$. Then there exists a compact set $K$ with $K \subset \Omega$, a continuous Radon measure of finite mass in $K, \mu_{\text {cont }}$, a countable subset $\mathfrak{S} \subset K$, and signed measures $\xi_{\mathbf{c}} \in \mathcal{M}(\mathbb{S})$ for $\mathbf{c} \in \mathfrak{S}$ such that (4.1) holds.

Let $\nu \in \mathcal{R}_{\mathrm{t}}^{\prime}(\Omega)$. Then there exists a compact set $K$ with $K \subset \Omega$, a continuous Radon measure of finite mass in $K, \mu_{\text {cont }}$, a countable subset $\mathfrak{S} \subset K$, signed measures $\xi_{\mathbf{c}} \in \mathcal{M}(\mathbb{S})$ and constants $\gamma_{\mathbf{c}} \in \mathbb{R}$ for $\mathbf{c} \in \mathfrak{S}$ such that (4.4) holds.

The spaces $\mathcal{R}_{\mathrm{c}}(\Omega)$ and $\mathcal{R}_{\mathrm{t}, \mathrm{c}}(\Omega)$ are the subspaces of $\mathcal{R}(\Omega)$ and $\mathcal{R}_{\mathrm{t}}(\Omega)$, respectively, formed by the functions with compact support in $\Omega$. As with $C_{\mathrm{c}}(\Omega)$, we give them the topology of the inductive limit of the spaces $\mathcal{R}[U]$ (or correspondingly $\mathcal{R}_{\mathrm{t}}[U]$ ) for $U$ a bounded open set with $\bar{U} \subset \Omega$ as $U \nearrow$. The elements of the dual spaces $\mathcal{R}_{\mathrm{c}}^{\prime}(\Omega)$ and $\mathcal{R}_{\mathrm{t}, \mathrm{c}}^{\prime}(\Omega)$ are measures with thick delta functions over $\Omega$ with finite variation over each bounded open set $U$ with $\bar{U} \subset \Omega$, but whose variation over the whole set $\Omega$ might be infinite. 


\section{Spaces of Regulated functions in one variable}

Our analysis of the spaces of regulated functions in several variables yields, of course, the corresponding analysis in one variable. In this section we describe the dual spaces in one variable and compare our results to those of other authors.

If $\phi$ is a regulated function of one variable, defined in $\mathbb{R}$, then $\phi$ is a normalized regulated function if

$$
\phi(x)=\frac{1}{2}(\phi(x+0)+\phi(x-0)),
$$

for each $x \in \mathbb{R}$. In one variable one may normalize in other ways, by asking, for instance, continuity from the right, or perhaps continuity from the left, and one can find such normalizations in the work of several authors.

We introduced the spaces $\mathcal{R}_{\mathrm{t}}[U]$ and $\mathcal{R}[U]$, if $U$ is a bounded open set. If $U=(a, b)$, a bounded interval, we obtain the spaces $\mathcal{R}_{\mathrm{t}}[(a, b)]$ of regulated functions defined in $\mathbb{R}$ which vanish outside of $[a, b]$ and its subspace $\mathcal{R}[(a, b)]$ consisting of the normalized regulated functions of $\mathcal{R}_{\mathrm{t}}[(a, b)]$.

It will be also convenient to introduce another space, the space $\mathcal{R}[a, b]$. A function $\phi \in \mathcal{R}_{\mathrm{t}}[(a, b)]$ belongs to $\mathcal{R}[a, b]$ if it is normalized at each point of $(a, b)$ but not necessarily at the endpoints. Similarly, we introduce the space $\mathcal{R}_{\mathrm{L}}[a, b]$, the subspace of $\mathcal{R}_{\mathrm{t}}[(a, b)]$ formed by those functions that are left continuous at each point of $\mathbb{R} \backslash\{a, b\}$. The spaces $\mathcal{R}[a, b]$ and $\mathcal{R}_{\mathrm{L}}[a, b]$ are clearly isomorphic, and we denote by $i: \mathcal{R}_{\mathrm{L}}[a, b] \longrightarrow \mathcal{R}[a, b]$ the isomorphism. One may want to think of the elements of $\mathcal{R}[a, b]$, or of $\mathcal{R}_{\mathrm{L}}[a, b]$, as regulated functions defined on $[a, b]$.

In one variable, thick delta functions are linear combinations of the one sided deltas, that is, expressions of the form $\xi_{+} \delta_{+}(x-c)+\xi_{-} \delta_{-}(x-c)$, where the right sided Dirac delta function at $x=c$ is the element $\delta_{+}(x-c)$ $=\delta(x-(c+0))$ of $\mathcal{R}^{\prime}[a, b]$ given by

$$
\left\langle\delta_{+}(x-c), \phi(x)\right\rangle=\phi(c+0)=\lim _{x \rightarrow c^{+}} \phi(x),
$$

and the left sided Dirac delta function at $x=c$ is the element $\delta_{-}(x-c)=$ $\delta(x-(c-0))$ of $\mathcal{R}^{\prime}[a, b]$ given by

$$
\left\langle\delta_{-}(x-c), \phi(x)\right\rangle=\phi(c-0)=\lim _{x \rightarrow c^{-}} \phi(x) .
$$

Observe that if a point $c$ is one of the endpoints, $a$ or $b$, then the Dirac delta functions $\delta(x-a)$ and $\delta(x-b)$, are actually a multiple of the one sided deltas in the space $\mathcal{R}^{\prime}[(a, b)]$,

$$
\delta(x-a)=\frac{1}{2} \delta_{+}(x-a), \quad \delta(x-b)=\frac{1}{2} \delta_{-}(x-b),
$$


but $\delta(x-a)$ and $\delta_{+}(x-a)$ are linearly independent in $\mathcal{R}^{\prime}[a, b]$, and so are $\delta(x-b)$ and $\delta_{-}(x-b)$.

It will be convenient, and it will simplify the notation, to think of $\delta_{-}(x-a)$ as $\delta(x-a)$ and of $\delta_{+}(x-b)$ as $\delta(x-b)$ when working in the space $\mathcal{R}^{\prime}[a, b]$.

If $\mu \in \mathcal{R}^{\prime}[a, b]$ then there exists a continuous Radon measure with support in $[a, b], \mu_{\text {cont }}$, and two functions $\xi_{+}$and $\xi_{-}$, defined in $\mathbb{R}$, and that vanish outside of a countable subset $\mathfrak{S}$ of $[a, b]$, such that

$$
\mu(x)=\mu_{\text {cont }}(x)+\sum_{c \in \mathfrak{S}}\left(\xi_{+}(c) \delta_{+}(x-c)+\xi_{-}(c) \delta_{-}(x-c)\right) .
$$

Furthermore, the norm of $\mu$ in the space $\mathcal{R}^{\prime}[a, b]$ is

$$
\|\mu\|=\int_{a}^{b} \mathrm{~d}\left|\mu_{\text {cont }}\right|+\sum_{c \in \mathfrak{S}}\left(\left|\xi_{+}(c)\right|+\left|\xi_{-}(c)\right|\right) .
$$

If $\nu \in \mathcal{R}_{\mathrm{t}}^{\prime}([a, b])$ then there exists a continuous Radon measure with support in $[a, b], \mu_{\text {cont }}$, and three functions $\xi_{+}, \xi_{-}$, and $\gamma$, defined in $\mathbb{R}$, and that vanish outside of a countable subset $\mathfrak{S}$ of $[a, b]$, such that

$$
\begin{aligned}
\nu(x)= & \mu_{\text {cont }}(x) \\
& +\sum_{c \in \mathfrak{S}}\left(\xi_{+}(c) \delta_{+}(x-c)+\xi_{-}(c) \delta_{-}(x-c)+\gamma(c) \delta(x-c)\right) .
\end{aligned}
$$

Moreover, the norm of $\nu$ in the space $\mathcal{R}_{\mathrm{t}}^{\prime}[a, b]$ is

$$
\|\nu\|=\int_{a}^{b} \mathrm{~d}\left|\mu_{\text {cont }}\right|+\sum_{c \in \mathfrak{S}}\left(\left|\xi_{+}(c)\right|+\left|\xi_{-}(c)\right|+|\gamma(c)|\right) .
$$

The spaces $\mathcal{R}(a, b)$ and $\mathcal{R}_{\mathrm{t}}(a, b)$ are the analogous of $C(a, b)$. Here $(a, b)$ is a general open interval, which may be bounded or not. A function $\phi$ belongs to $\mathcal{R}_{\mathrm{t}}(a, b)$ if it is regulated over $(a, b)$, without any restriction on its behavior at the endpoints; it belongs to $\mathcal{R}(a, b)$ if it is a normalized regulated function in $(a, b)$. The spaces $\mathcal{R}(a, b)$ and $\mathcal{R}_{\mathrm{t}}(a, b)$ are, like $C(a, b)$, topological vector spaces, but not Banach spaces: Convergence means uniform convergence over each compact subset of $(a, b)$. The dual space $(C(a, b))^{\prime}$ is the space of signed measures with compact support in $(a, b)$, and thus the dual spaces of $\mathcal{R}(a, b)$ and $\mathcal{R}_{\mathrm{t}}(a, b)$ are formed by signed measures with thick deltas and compact support. If $\mu \in \mathcal{R}^{\prime}[c, d]$, and $[c, d] \subset(a, b)$, then $\mu$ has a natural extension to $\mathcal{R}^{\prime}(a, b)$, which we shall denote with the same notation, $\mu$; similarly the elements of $\mathcal{R}_{\mathrm{t}}^{\prime}[c, d]$ have a natural extension to $\mathcal{R}_{\mathrm{t}}^{\prime}(a, b)$.

If $\mu \in \mathcal{R}^{\prime}(a, b)$, then there exists a closed interval $[c, d] \subset(a, b)$ such that $\mu \in \mathcal{R}^{\prime}[c, d]$. The form of $\mu$, a signed measure with thick delta functions, is given in (5.5). 
Let $\nu \in \mathcal{R}_{\mathrm{t}}^{\prime}(a, b)$. Then there exists a closed interval $[c, d] \subset(a, b)$ such that $\nu \in \mathcal{R}_{\mathrm{t}}^{\prime}[c, d]$. The form of $\nu$ is given in (5.7).

5.1. Comparison with other results in one variable. The dual spaces $\mathcal{R}_{\mathrm{t}}^{\prime}[(a, b)]$ and $\mathcal{R}^{\prime}[a, b]$ were described by Kaltenborn [8] in 1934, by using the Dushnik interior integral. According to his results, any $\nu \in \mathcal{R}_{\mathrm{t}}^{\prime}[(a, b)]$, it can be expressed as the integral

$$
\langle\nu, \phi\rangle=\int_{a}^{b} \phi \mathrm{d} \psi+\sum_{i=0}^{\infty}\left[\phi\left(c_{i}\right)-\phi\left(c_{i}-0\right)\right] \varphi\left(c_{i}\right),
$$

for $\phi \in \mathcal{R}_{\mathrm{t}}[(a, b)]$, where $\psi$ and $\varphi$ are two functions.

Let

$$
\zeta^{c}(x)=\left\{\begin{array}{l}
1, \text { when } x=c \\
0, \text { when } x \neq c .
\end{array}\right.
$$

We have that $\left\langle\nu, \zeta^{c}\right\rangle=\varphi(c)$. On the other hand, if $\nu$ has the expansion (5.7), we obtain $\left\langle\nu, \zeta^{c}\right\rangle=\gamma(c)$, so that

$$
\varphi(c)=\gamma(c) .
$$

Now let

$$
\lambda^{c}(x)=\left\{\begin{array}{cc}
0, & a \leq x<c \\
1 / 2, & x=c \\
1, & c<x \leq b
\end{array}\right.
$$

so that $\lambda^{c} \in \mathcal{R}[a, b]$. We have that

$$
\left\langle\nu, \lambda^{c}\right\rangle=\alpha(b)-\alpha(c+0)+\frac{1}{2}(\alpha(c+0)-\alpha(c-0)),
$$

where

$$
\alpha(x)=2 \psi(x)-\psi(x+0) .
$$

Notice that $\psi(x)$ is obtained by $\langle\nu(t), \kappa(t, x)\rangle$, where $\kappa(t, x)=1$ or 0 according as $a \leq t \leq x$ or $x<t \leq b$. Since (5.7) yields that $\left\langle\nu, \lambda^{c}\right\rangle=$ $\int_{c}^{b} \mathrm{~d} \mu_{\text {cont }}+\xi_{+}(c)$, we obtain

$$
\alpha(b)-\alpha(c+0)=\int_{c}^{b} \mathrm{~d} \mu_{\mathrm{cont}}
$$

and

$$
\frac{1}{2}(\alpha(c+0)-\alpha(c-0))=\xi_{+}(c) .
$$

Furthermore, Kaltenborn proved that any $\nu \in \mathcal{R}^{\prime}[a, b]$ can be represented as the Stieltjes mean integral,

$$
\langle\nu, \phi\rangle=(m) \int_{a}^{b} \phi \mathrm{d} \alpha,
$$

where (5.15) and (5.16) hold. 
It was shown by Tvrdý $[12,13,14]$ that the dual of $\mathcal{R}_{\mathrm{L}}[a, b]$, the space of left continuous regulated functions in $[a, b]$, can be described as follows. Each linear bounded functional $\Phi \in \mathcal{R}_{\mathrm{L}}^{\prime}[a, b]$ can be uniquely expressed by a pair $\eta=(p, q) \in \mathcal{B} \mathcal{V}[a, b] \times \mathbb{R}$, where $\mathcal{B} \mathcal{V}[a, b]$ are the functions of bounded variation on $[a, b]$, as

$$
\langle\Phi, \phi\rangle=\Phi_{\eta}(\phi)=q \phi(a)+\int_{a}^{b} p \mathrm{~d} \phi,
$$

for any $\phi \in \mathcal{R}_{\mathrm{L}}[a, b]$. The mapping

$$
\Phi: \eta \in \mathcal{B V}[a, b] \times \mathbb{R} \longmapsto \Phi_{\eta} \in \mathcal{R}_{L}^{\prime}[a, b],
$$

is an isomorphism $[12,13,14]$. The integral in $(5.18)$ is a Perron-Stieltjes integral, sometimes also called Henstock-Stieltjes integral.

If $i: \mathcal{R}_{\mathrm{L}}[a, b] \rightarrow \mathcal{R}[a, b]$ is the canonical isomorphism, and $\mu \in \mathcal{R}^{\prime}[a, b]$ then $\mu \circ i \in \mathcal{R}_{\mathrm{L}}^{\prime}[a, b]$, and each element $\Phi \in \mathcal{R}_{\mathrm{L}}^{\prime}[a, b]$ is of this form, namely, with $\mu=\Phi \circ i^{-1}$. If $\Phi=\Phi_{\eta}, \eta=(p, q) \in \mathcal{B} \mathcal{V}[a, b] \times \mathbb{R}$, and $\mu$ is given by (5.5) then if $\phi \in \mathcal{R}_{\mathrm{L}}[a, b]$,

$$
\langle\mu \circ i, \phi\rangle=\int_{a}^{b} \phi(x) \mathrm{d} \mu_{\mathrm{cont}}+\sum_{c \in \mathfrak{S}}\left(\xi_{+}(c) \phi(c+0)+\xi_{-}(c) \phi(c-0)\right) .
$$

Therefore,

$$
q=\left\langle\mu \circ i, \chi_{[a, b]}\right\rangle=\int_{a}^{b} \mathrm{~d} \mu_{\mathrm{cont}}+\xi_{+}(a)+\xi_{-}(b)
$$

while

$$
p(t)=\left\langle\mu \circ i, \chi_{(t, b]}\right\rangle, \quad t \in[a, b),
$$

so that

$$
p(t)=\int_{t}^{b} \mathrm{~d} \mu_{\text {cont }}+\sum_{c \in \mathfrak{S}, c<t}\left(\xi_{+}(c)+\xi_{-}(c)\right), \quad t \notin \mathfrak{S},
$$

and

$$
p(t)=\int_{t}^{b} \mathrm{~d} \mu_{\text {cont }}+\xi_{+}(t)+\sum_{c \in \mathfrak{S}, c<t}\left(\xi_{+}(c)+\xi_{-}(c)\right), \quad t \in \mathfrak{S} .
$$

When $t=b$ we obtain

$$
p(b)=\left\langle\mu \circ i, \chi_{[b]}\right\rangle=\xi_{+}(b) .
$$




\section{REFERENCES}

[1] C. Barnett and V. Camillo, Uniform limits of step functions, Math. Sci., 22 (1997), 65-68.

[2] S. K. Berberian, Regulated functions: Bourbaki's alternative to the Riemann integral, Amer. Math. Monthly, 86 (1979), 208-211.

[3] T. M. K. Davison, A generalization of regulated functions, Amer. Math. Monthly, 86 (1979), 202-204.

[4] J. Dieudonne, Foundations of Modern Analysis, Academic Press, New York, 1969.

[5] R. Estrada, The set of singularities of regulated functions of several variables, Collect. Math., 63 (2012), 351-359.

[6] R. Estrada and S. A. Fulling, Functions and distributions in spaces with thick points, Int. J. Appl. Math. Stat., 10 (2007), 25-37.

[7] T. H. Hildebrandt, On bounded linear functional operations, Trans. Amer. Math. Soc., 36 (1934), 868-875.

[8] H. S. Kaltenborn, Linear functional operations on functions having discontinuities of the first kind, Bull. Amer. Math. Soc., 40 (1934), 702-708.

[9] J. O'Donovan, Regulated functions on topological spaces, Real Anal. Exchange, 33 (2007-08), 405-416.

[10] E. Talvila, The distributional Denjoy integral, Real Anal. Exchange, 33 (2008), 51-82.

[11] E. Talvila, The regulated primitive integral, Illinois J. Math., 53 (2009), 1187-1219.

[12] M. Tvrdý, Regulated functions and the Perron-Stieltjes integral, Casopis Pěst. Mat., 114 (1989), 187-209.

[13] M. Tvrdý, Linear bounded functionals on the space of regular regulated functions, Tatra Mt. Math. Publ., 8 (1996), 203-210.

[14] M. Tvrdý, Differential and integral equations in the space of regulated functions, Mem. Differential Equations Math. Phys., 25 (2002), 1-104.

[15] J. Vindas and R. Estrada, Distributionally regulated functions, Studia Math., 181 (2007), 211-236.

[16] J. Vindas and R. Estrada, On the jump behavior of distributions and logarithmic averages, J. Math. Anal. Appl., 347 (2008), 597-606.

(Received: June 4, 2012)

\author{
Mathematics Department \\ Louisiana State University \\ Baton Rouge, LA 70803 \\ U.S.A. \\ yyang18@math.lsu.edu \\ restrada@math.lsu.edu
}

